Relations industrielles

Industrial Relations

\title{
G.W. ADAMS : Canadian Labour Law - A Comprehensive Text. Aurora, Canada Law Book Inc., 1985, 983 pp., ISBN 0-888-04-030-X
}

\section{Pierre Verge}

Volume 41, numéro 3, 1986

URI : https://id.erudit.org/iderudit/050244ar

DOI : https://doi.org/10.7202/050244ar

Aller au sommaire du numéro

Éditeur(s)

Département des relations industrielles de l'Université Laval

ISSN

0034-379X (imprimé)

1703-8138 (numérique)

Découvrir la revue

Citer ce compte rendu

Verge, P. (1986). Compte rendu de [G.W. ADAMS : Canadian Labour Law - A Comprehensive Text. Aurora, Canada Law Book Inc., 1985, 983 pp., ISBN 0-888-04-030-X]. Relations industrielles / Industrial Relations, 41(3), 659-661. https://doi.org/10.7202/050244ar

Tous droits réservés @ Département des relations industrielles de l'Université Laval, 1986
Ce document est protégé par la loi sur le droit d'auteur. L’utilisation des services d'Érudit (y compris la reproduction) est assujettie à sa politique d'utilisation que vous pouvez consulter en ligne.

https://apropos.erudit.org/fr/usagers/politique-dutilisation/ 
Les résumés qui apparaissent en ce tome II sont de même confection que ceux déjà publiés dans les recueils antérieurs. Il s'agit de résumés succints qui présentent l'essentiel des positions patronale et syndicale à l'arbitrage ainsi que l'essence de la décision arbitrale. Ces résumés ne sont pas toujours une source sûre pour assurer une compréhension adéquate de la portée de la décision, en ce sens que le droit régissant alors les parties ainsi que les faits ne sont pas suffisamment particularisés; il faut donc, après cette première démarche qui permet de faire une collecte rapide de la matière susceptible d'intérêt, consulter les sentences.

En définitive, le service offert par Coplanam est honnête et ne manque pas d'intérêt. Il facilite indéniablement la collecte des données. Il ne faudrait surtout pas croire que cet instrument de travail peut cependant fournir une réponse complète à un problème particulier. D'autant plus que l'arbitrage des griefs évolue depuis quelques années à une cadence effrénée et que les besoins des utilisateurs se font maintenant surtout sentir en termes d'informations au jour le jour.

Rodrigue BLOUIN

Université Laval

Canadian Labour Law - A Comprehensive Text, par G.W. Adams, Aurora, Canada Law Book Inc., 1985, 983 pp., ISBN 0-888-04-030-X

L'impressionnant ouvrage du professeur Adams, qui fut pendant près de cinq ans président du Ontario Labour Relations Board, se veut, pour reprendre les mots mêmes de la préface, un traité de droit du travail d'envergure pancanadienne ( $«$... a text on Labour Law written from an over-all Canadian perspective»). De l'exposé des différentes lois canadiennes du travail, ainsi que de la jurisprudence pertinente, devraient, compte tenu de bien des particularismes, ressortir certains traits dominants caractéristiques de tout cet ensemble: «It is my hope that this book will help identify the 'sameness' in Canadian Labour law and facilitate a greater awareness of the contribution each jurisdiction is making to the development of Canadian Labour law» (p. V). Il semblerait indiqué de dissiper d'emblée deux ambiguités de taille par rapport à la réalisation de l'objectif ainsi fixé, de même qu'en définitive, par rapport à sa présentation, à son titre: Canadian Labour Law. Même si cette démarche initiale risque de paraître injustement négative face à une oeuvre dont l'ampleur et la précision font naître assez spontanément l'éloge, elle aura à tout le moins, l'avantage d'appeler un chat, un chat. L'ouvrage bien identifié, ses qualités certaines n'en seront alors que plus manifestes.

En premier lieu, l'auteur fait le tour de l'encadrement juridique de ce qu'il est assez bien convenu d'appeler les rapports collectifs du travail. Il se confine toutefois à ceux-ci, à l'exclusion de l'étude de la situation juridique de la majorité des salariés qui se situent à l'extérieur de ce cadre. En d'autres termes, il s'agit d'un ouvrage qui traite du contenu usuel de «Labour Relations Law», mais non d'«Employment Law». À cet égard, le titre de l'ouvrage, quelque peu ancien déjà, de Carrothers (Collective Bargaining Law in Canada) était plus exact. Le vocable "droit du travail», qui rend bien «labour law», inclut, tant d'un point de vue théorique que pratique, l'un et l'autre des deux sujets précédents. Constatons de surcroît l'amplification de l'intervention législative et le renouveau en partie du droit touchant l'ensemble du salariat au cours des dernières années et de façon générale au pays (prohibition de la discrimination dans l'emploi, santé et sécurité du travail, protection à l'encontre de différents types de licenciement...).

En second lieu, malgré la présence de certains éléments pertinents, l'ouvrage, du moins dans une véritable perspective juridique scientifique, se confine au monde des provinces cana- 
diennes de Common Law, à l'exclusion du Québec. Certes, l'auteur situe les différents textes du Code du travail du Québec au nombre des passages comparables des dix autres lois canadiennes des rapports du travail. Il lui arrive même, quoique non méthodiquement, de citer à l'occasion quelques décisions ou jugements s'y rapportant (le traitement des effets de l'aliénation de l'entreprise sur l'accréditation et la convention collective paraît ainsi adéquat; on ne pourrait cependant, par exemple, en dire autant de celui relatif à l'obligation de représentation égale, du moins au regard de la jurisprudence des instances québécoises, assez circonstanciées pourtant en la matière). Surtout, il faut lui savoir gré de bien dégager les principales écoles législatives, par rapport aux grands thèmes abordés par ces différentes lois. Le lecteur trouvera également une synthèse fort réussie quant à l'évolution et au contenu présent du régime particulier relatif à l'industrie de la construction au Québec. Toutefois, sans parler d'une omission quasi-totale de la doctrine québécoise pertinente, force est d'en venir à la constatation essentielle: tout l'arrière-plan juridique est exclusivement celui de la Common-Law. L'«autre» droit commun canadien, du moins pour ce qui est du droit privé, qui est ici en cause le plus souvent, le système de droit civil, qui, dans une perspective universelle, - est-il besoin de le dire? - se rattache à l'un des grands ensembles juridiques contemporains, est absent. Comment alors, sur le plan de la science juridique, prétendre à une oeuvre «canadienne»? Certes, pourra-t-on rétorquer, après tout, les résultats pratiques ne diffèrent pas tellement d'un système à l'autre; ou encore, ce droit commun - l'un ou l'autre - est-il si important, face à des lois du travail qui y dérogent d'une façon aussi marquée? Il n'en demeure pas moins que le droit commun (comme en témoignent précisément les importants développements de Common Law dans l'ouvrage) reste fort pertinent relativement à la discussion de la nature de l'institution syndicale, au traitement de situations illégales de grève, de piquetage, de lockout et de boycottage ... Or, à chacune de ces occasions, une analyse civiliste est tout aussi indiquée qu'une analyse selon la méthodologie propre à la Common Law, sous réserve, bien entendu, de la portée de la législation qui déroge au droit commun. Inutile d'insister: le présent ouvrage, simplement, $s$ 'il veut prétendre à l'appellation "Canadian» sur le plan juridique, souffre de la même carence scientifique que d'autres volumes, pourtant également excellents sous bien d'autres rapports, tels celui de Carrothers, précité, ou encore, celui, plus récent, de Arthurs, Carter et Glasbeek, Labour Law and Industrial Relations in Canada.

Envisagé maintenant, sous son jour véritable, comme un traité de droit des rapports collectifs du travail véhiculant un arrière-plan de Common Law, nous sommes en présence d'une oeuvre fort documentée, particulièrement sur le plan jurisprudentiel. Dans un style clair, on nous entraîne ainsi, après des synthèses préliminaires traitant des origines des différentes lois canadiennes et de la portée du partage constitutionnel de la compétence législative, vers l'examen particulier du contenu usuel de ces différentes lois, l'accent étant généralement mis sur les lois fédérale, d'Ontario et de la Colombie-Britannique. Le point de départ, qui trahit soit dit en passant, une mentalité juridique parmi d'autre, n'est pas le droit matériel. La préoccupation initiale est, en effet, institutionnelle, reliée à la mise en oeuvre de la règle juridique. Il s'agit du fonctionnement et des pouvoirs de l'organisme typiquement chargé d'administrer les principaux aspects de la loi: le conseil des relations du travail. Qui plus est, ce chapitre consacré aux Boards est lui-même précédé d'un autre décrivant l'exercice du contrôle judiciaire de l'activité de ces organismes ainsi que des tribunaux d'arbitrage. Le particularisme du Code de procédure civile du Québec, qui se conjugue ici à l'occasion aux principes fondamentaux issus de la Common Law n'apparaît pas, soit dit en passant. Etablissement, ensuite, dans d'autres chapitres, de l'identité, du statut juridique des personnes et organismes visés par la loi, modes de détermination du représentant collectif des salariés - avec insistance particulière sur la définition de l'unité de négociation; incidence sur ce statut de l'aliénation ou de la concession de l'entreprise; perte de ce statut; description des différentes pratiques déloyales, portée en particulier de l'obligation fondamentale de négocier de bonne foi et description détaillée des pouvoirs répara- 
teurs dont jouissent les différents conseils de relations du travail. Le «conflit industriel» est aussi l'objet d'un chapitre distinct, dans lequel, avons-nous vu, le droit commun se conjugue aux dispositions de la loi pour établir les paramètres de la légalité de situations de grève, de lockout, de boycottage et de piquetage. Les aspects usuels du régime juridique de la convention collective - du moins celle de type usuel, liant les «salariés», au sens de la loi, et dont la force obligatoire provient de celle-ci - sont ensuite étudiés, y compris ceux de l'arbitrage des griefs, qui assure sa mise en oeuvre. Des chapitres distincts sont enfin consacrés à l'obligation de représentation égale, qui incombe à l'agent négociateur, de même qu'aux différents aspects juridiques de la vie des syndicats, y compris la problématique de leur personnalité juridique ainsi qu'aux syndicats de métiers et à l'industrie de la construction.

Le simple énoncé de ces titres de chapitres ne saurait qu'être une invitation adressée au lecteur à fréquenter le contenu, riche et détaillé, de l'ouvrage. À titre illustratif, mais aucunement limitatif, je signalerais, par exemple, ces pages particulièrement éclairantes portant sur l'incidence de la transformation ou de la fermeture de l'entreprise sur les rapports collectifs, dans lesquels les différentes solutions canadiennes - y compris, avons-nous vu, dans ce cas, celles se dessinant au Québec à partir de l'article 45, C.t. - sont appréciées à la lumière du droit américain. D'un intérêt tout particulier également, ces développements relatifs à la liberté d'expression de l'employeur et à l'obligation de négocier de bonne foi, où, là aussi, les rapprochements avec les solutions américaines sont enrichissantes. Signalons aussi, quant au contenu de la convention collective, une bonne discussion de la portée des diverses clauses de sécurité syndicale; on observe toutefois un silence relatif en ce qui a trait au contenu obligationnel implicite de cette convention. L'analyse du statut juridique du syndicat véhicule l'ensemble de l'évolution jurisprudentielle de Common Law, tant en Angleterre qu'au pays; il y est aussi fait mention de l'adoption des textes du Code de procédure civile québécois traitant de la capacité d'ester en justice de ce type de groupement. L'exposé des paramètres s'imposant à l'exercice de la discipline syndicale reflète bien les principes fondamentaux du droit; il eut été possible, en des situations d'expulsion du membre de son syndicat en particulier, d'insister plus fortement sur l'incidence de la transformation de la nature de l'instance syndicale sous l'effet du rôle de représentation obligatoire dont le charge la loi des rapports du travail contemporain.

Aux endroits stratégiques - portée, par exemple, de la représentation collective obligatoire des salariés, l'effet des clauses de sécurité syndicale, liberté d'expression de l'employeur, celle du syndicat à l'occasion de situation de piquetage ... - l'auteur formule les interrogations présentes, quant à l'incidence de la Charte des droits et libertés de la personne; des rapprochements, nuancés sont alors faits avec les grandes solutions américaines. En revanche, le contenu du droit international pertinent est, dans l'ensemble, absent.

Dans sa totalité, l'ouvrage dont nous avons déjà signalé l'ampleur documentaire et la clarté de l'exposé, témoigne d'une grande familiarité avec la pratique des relations du travail. Il faut $y$ voir, sans contexte, une contribution majeure au développement du droit du travail canadien.

Pierre VERGE

Université Laval 